\title{
THE TINSLEY DIAGRAM REVISITED
}

\author{
Stephen Leonard and Kayll Lake ${ }^{1}$ \\ Department of Physics, Queen's University at Kingston, Kingston, \\ Ontario, Canada K7L 3N6
}

Received __; accepted _

\footnotetext{
${ }^{1}$ E-mail: lake@astro.queensu.ca
} 


\begin{abstract}
Motivated by the recent determinations of the Hubble constant $\left(H_{0}\right)$ from observations of Cepheid variables in NGC4571 and M100, we plot the Tinsley diagram with level curves of the cosmological constant $(\Lambda)$. Based on current estimates of the absolute ages of globular clusters we conclude that $\Lambda>0$ and, irrespective of the background spatial curvature, the universe will not recollapse. These conclusions hold for both relativistic and Newtonian models and are independent of the density parameter.
\end{abstract}

Subject headings: cosmology, theory 


\section{Introduction}

Tinsley's modification of the Robertson diagram (Tinsley (1967)), the plot of $H_{0} t_{0}$ vs. $\log \left(\Omega_{0} / 2\right)$, where $t_{0}$ is the age of the universe and $\Omega_{0}$ the current density parameter, allows us to limit the cosmological parameters simply because $t_{0}$ must be greater than the oldest observed objects. (See, for example Rindler (1977) for an introduction.) In view of the recent determinations of $H_{0}$ from the direct observations of Cepheid variables in NGC4571 (Pierce et al. (1994)) and M100 (Ereedman et al. (1994)] in the Virgo cluster it would seem an appropriate time to reexamine the Tinsley diagram. In this Letter we construct the diagram based on a relativistic model consisting of a non-interacting mixture of dust and blackbody radiation. (This is known to be an excellent approximation to a baryon-conserving equilibrium mixture of blackbody photons and a neutral relativistic Maxwell-Boltzmann gas for an initial $f\left(\equiv n \gamma / n_{b}\right.$, photon to baryon number density ratio $)>$ 100 which is most certainly the case. Other (non-baryonic) species are handled analogously.)

\section{Construction of the Diagram}

\subsection{Relativistic model}

Friedmann's equation for the evolution of a separately conserved distribution of dust and blackbody radiation gives rise to the relation

$$
h t_{0} \propto \int_{0}^{1} \frac{w d w}{\sqrt{\lambda w^{4}+(1-\lambda) w^{2}-\left(\Omega_{0}+\tau_{0}\right) w^{2}+\Omega_{0} w+\tau_{0}}}
$$

where $h, \lambda, \Omega_{0}$, and $\tau_{0}$ are dimensionless parameters. We measure $t_{0}$ in units of 10 Gyr. The proportionality constant turns out to be $1 / 1.02$. (In what follows we display three 
significant figures). The parameters are defined by

$$
h=H_{0} / 100
$$

where $H_{0}$ is in $\mathrm{km} \mathrm{s}^{-1} \mathrm{Mpc}^{-1}$, and

$$
\lambda=2.8510^{55} \Lambda / h^{2}
$$

where $\Lambda$ is in $\mathrm{cm}^{-2} \cdot \tau_{0}$ is defined by

$$
\tau_{0}=32 \pi G c \sigma T_{0}^{4} / 3 H_{0}^{2}
$$

where $\sigma$ is the Stephan-Boltzmann constant, $G$ is Newton's constant, $T_{0}$ is the current background temperature, and $c$ is the speed of light. We find

$$
\tau_{0}=2.3810^{-5} / h^{2}
$$

$\Omega_{0}$ is defined by

$$
\Omega_{0}=320 \zeta(3) G T_{0}^{3}\left(m_{e}+m_{p}\right) \sigma / \pi^{3} f c k H_{0}^{2}
$$

where $\zeta$ is Riemann's function, $k$ is Boltzmann's constant, and $m_{e}$ and $m_{p}$ give the electron and proton masses. We find

$$
\Omega_{0}=3.5610^{7} / f h^{2}
$$

The spatial curvature of the background is determined by $\epsilon$ where

$$
\operatorname{sign}(\epsilon)=\operatorname{sign}\left(\Omega_{0}+\tau_{0}+\lambda-1\right)
$$

We have relegated the deceleration parameter $q_{0}$ to a supplementary role. It is determined by the relation

$$
q_{0}=\Omega_{0} / 2+\tau_{0}-\lambda
$$

For other number-conserving massive particle species which can be treated as dust, we replace $f$ by $n_{\gamma} / n$ and $m_{e}+m_{p}$ by $m$ with $n \equiv \Sigma_{s} n_{s}$ and $m \equiv \Sigma_{s} m_{s}$ where we sum over all particle species. Whereas the inclusion of non-baryonic species changes the interpretation of $f$ and the numerical coefficient in relation (7), the actual value of $\Omega_{0}$ does not enter the arguments which follow. 


\subsection{Newtonian model}

It turns out that $\tau_{0}$ makes no significant contribution to the integral (1) for $\log _{10}\left(\Omega_{0} / 2\right)>-2.5$ (the range we consider). With $\tau_{0}=0$ the integral (1) can be obtained directly from a Newtonian model with the Poisson equation generalized to read as

$$
\nabla^{2} \Phi+c^{2} \Lambda=4 \pi G \rho
$$

The integral (1) follows from the integral of the energy along with the equation of continuity. In this model

$$
\Omega_{0}=5.3210^{28} \rho_{0} / h^{2}
$$

where $\rho_{0}$ is the Newtonian mass density in $\mathrm{g} \mathrm{cm}^{-3}$ and includes all species contributing to the "dust".

\section{The Diagram}

\subsection{Properties}

Numerical integration of the integral (1) results in Figure 1. The ordinate $y$ is $1.02 h t_{0}$ and the abscissa $\log _{10}\left(\Omega_{0} / 2\right)$ where $\Omega_{0}$ is interpreted by relation $(7)$ or $(11)$. The range in $x$ shown covers all possibilities as $x<-2.3$ violates the primordial production of deuterium and ${ }^{3}$ He. Note that $\Omega_{0}=1$ corresponds to $x=-0.30$. Level curves of constant $\lambda$ are shown as is the locus $\epsilon=0$. It is important to note that models which recollapse lie below $\Lambda=0$ up to its intersection with $\epsilon=0$ and below the locus

$$
27 \lambda \Omega_{0}^{2}=4\left(1-\lambda-\Omega_{0}-\tau_{0}\right)^{3}
$$

thereafter. For the range shown the locus (12) lies close to $\Lambda=0$. 


\subsection{Limits}

Clearly $y>1.02 h t^{*} \equiv y^{*}$ where $t^{*}$ corresponds to the oldest absolute age observed.

Given $h$, this may be viewed as an absolute bound on $\lambda$ and $\epsilon$ over the range of $x$ shown. We choose to determine $t^{*}$ by the absolute ages of globular clusters.

According to Pierce et al. (1994)

$$
h=0.87 \pm 0.07
$$

and according to Freedman et al. (1994)

$$
h=0.80 \pm 0.17
$$

Chaboyer (1994) (see also Chaboyer et al. (1994)) gives

$$
t^{*}=1.55 \pm 0.4
$$

whereas VandenBergh (1991) gives

$$
t^{*}=1.65 \pm 0.2
$$

From the observations (13) and (14) with the ages (15) and (16) we find

$$
y^{*}=1.36 \pm 0.36
$$

where we have weighted all opinions equally and quoted the standard deviation associated with the sixteen extreme values. From Figure 1 and the estimate (17) we obtain the following conclusions:

i) The universe will not recollapse,

and

ii) $\Lambda>0$.

It should be noted that conclusion i) holds even if $\epsilon>0$. 


\section{Discussion}

It is common knowledge that a high value of $h$, given the ages of globular clusters, would lead to $\Lambda>0$. The purpose of this Letter is to make the argument, which is independent of the value of $\Omega_{0}$, clear. There is a well known resistance to $\Lambda$ on both aesthetic and philosophical grounds, the latter being encapsulated by the argument that $\Lambda$ affects all matter but is affected by nothing (for example, Coles and Ellis (1994)). However, one could also argue, on the basis of the Weyl-Lovelock theorem (Lovelock (1971)], that $\Lambda$ is an essential element of the underlying theory. This theorem states that in four dimensions the most general symmetric tensor field $A_{i j}=0$ which is divergence free and constructed out of the metric tensor and its first two (partial) derivatives is $A_{i j}=G_{i j}+\Lambda g_{i j}$. We are no more justified in dismissing $\Lambda$ on the basis of its size $\left(\sim\right.$ few $\left.10^{-56} \mathrm{~cm}^{-2}\right)$ than we are in dismissing quantum mechanics because of the value of Planck's constant. However, if $\Lambda>0$, as the empirical evidence would suggest, a number of fundamental results in general relativity (including the uniqueness theorems) will have to be reexamined.

We thank Brian Chaboyer for the estimate (15) and Dave Hanes for a preprint of Freedman et al. 1994. 


\section{REFERENCES}

Chaboyer, B. 1994, private communication

Chaboyer, B. Demarque, P., Guenther, D. B., Pinsonneault, M. H., \& Pinsonneault, D. B. 1994, preprint

Coles, P. \& Ellis, G. 1994, preprint

Freeman, W. L. et al. 1994 Nature, 371, 757

Lovelock, D. 1971, J. Math. Phys., 12, 498

Pierce, M. J. et al. 1994 Nature, 371, 385

Rindler, W. 1977, Essential Relativity (New York: Springer-Verlag)

Tinsley, B. M. 1967, Ph.D. dissertation, University of Texas

VandenBergh, D. A. 1991, in The Formation and Evolution of Star Clusters, PASP Conf. Ser. 13 ed.K. Janes (San Francisco:Astr. Soc. Pacific), 183

Note added in proof.- Accoring to X. Shi (private communication, and preprint [1994]) a high helium abundance (e.g. $Y \approx 0.28$ ), as well as helium diffusion and oxygen-enhancement in stellar models could lower $t^{*}$ to about $1.1 \pm 0.1$. With the observations (13) and (14) this would lower $y^{*}$ to $0.92 \pm 0.18$.

This manuscript was prepared with the AAS LATEX macros v3.0.

Fig. 1.- The Tinsley diagram plotted with level curves of constant $\lambda$. The curves shown correspond to $\lambda=-3,-2,-1,0,1,2,3,3.5,4$, and 5 . The locus $\epsilon=0$ is also shown. 


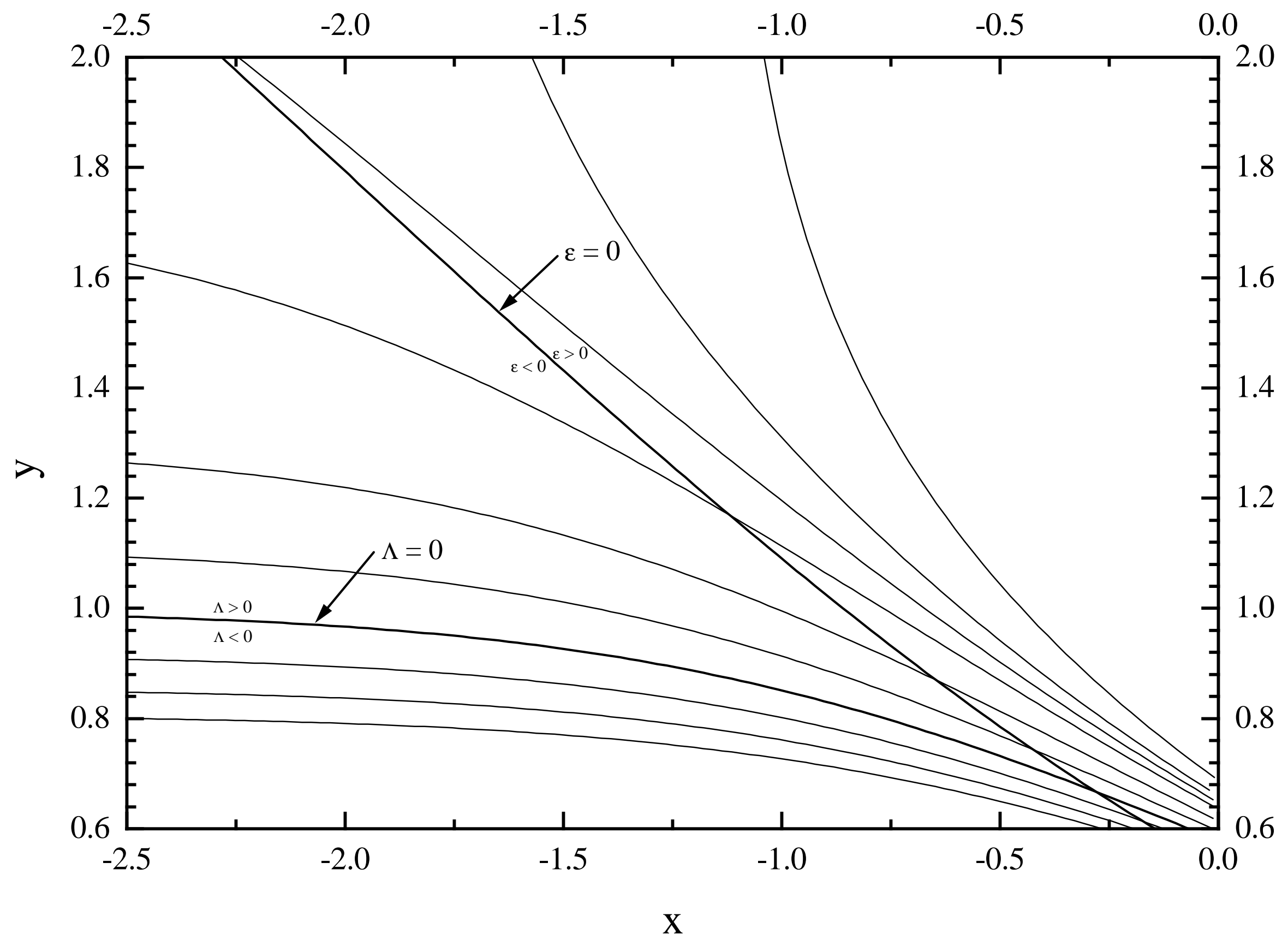

